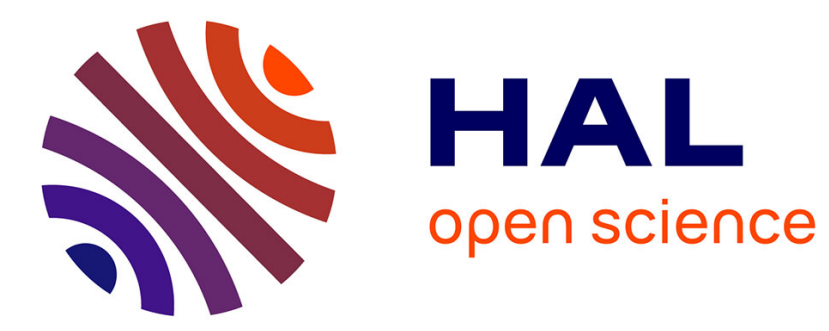

\title{
Sensorless Fault Tolerant Control Based On Backstepping Strategy For Induction Motors
}

Nadia Djeghali, Malek Ghanes, Jean-Pierre Barbot, Said Djennoune

\section{To cite this version:}

Nadia Djeghali, Malek Ghanes, Jean-Pierre Barbot, Said Djennoune. Sensorless Fault Tolerant Control Based On Backstepping Strategy For Induction Motors. IFAC WC, Aug 2011, Milan, Italy. hal00654250

\section{HAL Id: hal-00654250 \\ https://hal.inria.fr/hal-00654250}

Submitted on 21 Dec 2011

HAL is a multi-disciplinary open access archive for the deposit and dissemination of scientific research documents, whether they are published or not. The documents may come from teaching and research institutions in France or abroad, or from public or private research centers.
L'archive ouverte pluridisciplinaire HAL, est destinée au dépôt et à la diffusion de documents scientifiques de niveau recherche, publiés ou non, émanant des établissements d'enseignement et de recherche français ou étrangers, des laboratoires publics ou privés. 


\title{
Sensorless Fault Tolerant Control Based On Backstepping Strategy For Induction Motors
}

\author{
N. DJEGHALI ${ }^{*}$, M. GHANES ${ }^{* *}$, J. P. BARBOT ${ }^{* *}$, \\ S. DJENNOUNE* \\ * Laboratoire de Conception et Conduite des Systèmes de Production, \\ Université Mouloud MAMMERI de Tizi-Ouzou, B.P.17, 15000, \\ Algérie \\ ${ }^{* *}$ ECS-Lab, ENSEA, 6 avenue du Ponceau, 95014 Cergy, France
}

\begin{abstract}
In this paper, a fault tolerant control for induction motors based on backstepping strategy is designed. The proposed approach permits to compensate both the rotor resistance variations and the load torque disturbance. Moreover, to avoid the use of speed and flux sensors, a second order sliding mode observer is used to estimate the flux and the speed. The used observer converges in a finite time and permits to give a good estimate of flux and speed even in presence of rotor resistance variations and load torque disturbance. The simulation results show the efficiency of the proposed control scheme.
\end{abstract}

\section{INTRODUCTION}

Induction Motors (IM) are widely used in many industrial processes due to their reliability, low cost and high performance. However, because of several stresses (mechanical, environmental, thermal, electrical), IM are subjected to various faults, such as stator short-circuits and rotor failures such as broken bars or rings,...etc. The diagnostic of IM has shown that the presence of faults leads to parameters variations (Moreau et al. [1999]). In this work, we focus on the rotor resistance variations.

Fault Tolerant Control (FTC) systems are able to maintain specific systems performances not only under nominal conditions but also when faults occur (change in system parameters or characteristic properties). There are two types of FTC: active and passive approaches. In the passive approach, the controller is designed to maintain acceptable performances against a set of faults without any change in the control law. In the active approach, first the faults are detected and isolated (fault detection and isolation step), second the control law is changed (control reconfiguration step) to maintain specific performances (blanke et al. [2003], blanke et al. [2001]). This paper is concerned with the passive fault tolerant controller for IM in order to compensate the rotor resistance variations and the load torque disturbance. The proposed approach uses a direct field oriented controller based on backstepping strategy to steer the flux and the speed to their desired references in presence of rotor resistance variations and load torque disturbance. Moreover, sensorless control is considered. This control method avoids the use of the speed sensor (Holtz [2006], Ghanes et al. [2009], Ghanes et al. [2010]). For instance, in Ghanes et al. [2010] the feedback controller uses an adaptive observer in order to estimate the flux and the speed, in Ghanes et al. [2009] the control scheme is based on a first order sliding mode observer. The sliding mode observers are widely used due to their finite time convergence, robustness with respect to uncertainties and the possibility of uncertainty estimation (Perruqueti et al. [2002], Edwards et al. [2000]). When we use the first order sliding mode approach the chattering effect appears. To avoid the chattering effect, the high order sliding mode techniques have been developed. In this work, the controller uses a second order sliding mode observer (Solvar et al. [2010], Levant [1998], Floquet et al. [2007]) to estimate the speed and the flux.

Compared to the existing fault tolerant control schemes reported in the literature (Diallo et al. [2004], Bonivento et al. [2004], Djeghali et al. [2010], Tahami et al. [2006], Fekih [2008]) the contribution of this paper is first the design of a backstepping controller in presence of rotor resistance variations and load torque disturbance and second is the estimation of the speed by a second order sliding mode observer which uses only the measured stator currents.

This paper is organized as follows: Section 2 describes the IM oriented model in presence of rotor resistance variations. Section 3 gives some definitions on the practical stability. Section 4 is devoted to the design of the backstepping controller which is able to steer the flux and speed variables to their desired references in presence of rotor resistance variations and load torque disturbance. Section 5 presents the speed and flux estimation by using a second order sliding mode observer. Section 6 shows the simulation results. Section 7 gives some concluding remarks on the proposed fault tolerant controller.

\section{INDUCTION MOTOR ORIENTED MODEL}

In field oriented control, the flux vector is forced on the $\mathrm{d}$-axis $\left(\phi_{q r}=\frac{d \phi_{q r}}{d t}=0\right)$. The resulting induction motor model in the $(d-q)$ reference frame is described by the following state equations (Canudas [2000]): 


$$
\begin{aligned}
\frac{d i_{d s}}{d t} & =-a i_{d s}+\omega_{s} i_{q s}+\frac{L_{m}}{\sigma L_{s} L_{r} \tau_{r}} \phi_{d r}+\frac{V_{d s}}{\sigma L_{s}} \\
\frac{d i_{q s}}{\mathrm{~d} t} & =-a i_{q s}-\omega_{s} i_{d s}-\frac{L_{m}}{\sigma L_{s} L_{r}} P \Omega \phi_{d r}+\frac{V_{q s}}{\sigma L_{s}} \\
\frac{d \phi_{d r}}{d t} & =\frac{L_{m}}{\tau_{r}} i_{d s}-\frac{1}{\tau_{r}} \phi_{d r} \\
\frac{d \Omega}{d t} & =\frac{P L_{m}}{L_{r} J} i_{q s} \phi_{d r}-\frac{f}{J} \Omega-\frac{T}{J}
\end{aligned}
$$

with:

$$
\begin{gathered}
\omega_{s}=P \Omega+\frac{L_{m}}{\tau_{r} \phi_{d r}} i_{q s} \\
a=\left(\frac{R_{s}}{\sigma L_{s}}+\frac{1-\sigma}{\sigma \tau_{r}}\right)
\end{gathered}
$$

Where $\sigma$ is the coefficient of dispersion, given by:

$$
\sigma=1-\frac{L_{m}^{2}}{L_{s} L_{r}}
$$

$L_{s}, L_{r}, L_{m}$ are stator, rotor and mutual inductance, respectively. $R_{s}, R_{r}$ are respectively stator and rotor resistance. $\omega_{s}$ is the stator pulsation. $\tau_{r}$ is the rotor time constant $\left(\tau_{r}=\frac{L_{r}}{R_{r}}\right) . P$ is the number of pole pairs. $V_{d s}$, $V_{q s}$ are stator voltage components. $\phi_{d r}, \phi_{q r}$ are the rotor flux components. $\Omega$ is the mechanical speed. $T$ is the load torque. $i_{d s}, i_{q s}$ are stator current components. $J$ is the moment of inertia of the motor. $f$ is the friction coefficient. In presence of rotor resistance variations, the model (1) becomes:

$$
\begin{aligned}
\frac{d i_{d s}}{d t} & =-a i_{d s}+\omega_{s} i_{q s}+\frac{L_{m}}{\sigma L_{s} L_{r} \tau_{r}} \phi_{d r}+\frac{V_{d s}}{\sigma L_{s}}+h_{1}(x) \\
\frac{d i_{q s}}{d t} & =-a i_{q s}-\omega_{s} i_{d s}-\frac{L_{m}}{\sigma L_{s} L_{r}} P \Omega \phi_{d r}+\frac{V_{q s}}{\sigma L_{s}}+h_{2}(x) \\
\frac{d \phi_{d r}}{d t} & =\frac{L_{m}}{\tau_{r}} i_{d s}-\frac{1}{\tau_{r}} \phi_{d r}+h_{3}(x) \\
\frac{d \Omega}{d t} & =\frac{P L_{m}}{L_{r} J} i_{q s} \phi_{d r}-\frac{f}{J} \Omega-\frac{T}{J}
\end{aligned}
$$

where $x=\left(i_{d s}, i_{q s}, \phi_{d r}, \Omega\right) . h_{1}(x), h_{2}(x), h_{3}(x)$ represent the fault terms due to rotor resistance variations, they are given by:

$$
\begin{gathered}
h_{1}(x)=\Delta R_{r}\left(-\left(\frac{1-\sigma}{\sigma L_{r}}\right) i_{d s}+\frac{L_{m}}{\phi_{d r} L_{r}} i_{q s}^{2}+\frac{L_{m}}{\sigma L_{s} L_{r}^{2}} \phi_{d r}\right) \\
h_{2}(x)=\Delta R_{r}\left(-\left(\frac{1-\sigma}{\sigma L_{r}}\right) i_{q s}-\frac{L_{m}}{\phi_{d r} L_{r}} i_{d s} i_{q s}\right) \\
h_{3}(x)=\Delta R_{r}\left(\frac{L_{m}}{L_{r}} i_{d s}-\frac{\phi_{d r}}{L_{r}}\right)
\end{gathered}
$$

Here we introduce some definitions on the practical stability which will be used in the next section.

\section{PRELIMINARY}

Consider the following system:

$$
\begin{aligned}
\dot{x} & =f(t, x) \\
x\left(t_{0}\right) & =x_{0}, t_{0} \geq 0
\end{aligned}
$$

where $x \in R^{n}, t \in R_{\geq 0}$ and $f: R_{\geq 0} \times R^{n} \rightarrow R^{n}$ is piecewise continuous in $t$ and locally Lipschitz in $x$. $\left(t_{0}, x_{0}\right)$ are the initial conditions. We introduce the following definition in which $B_{r}$ denotes the closed loop ball in $R^{n}$ of radius $r$ centered at the origin, i.e: $B_{r}=\left\{x \in R^{n}\right.$ : $\|x\| \leq r\}$, with $\|$.$\| denotes the Euclidean norm of vectors.$
Definition 1. The system (4) is said to be globally uniformly exponentially pratically stable (or convergent to a ball $B_{r}$ with radius $r>0$ ), if there exists $\beta>0$, such that for all $t_{0} \in R_{\geq 0}$ and all $x_{0} \in R^{n}$ there exists $k \geq 0$ such that $\|x\| \leq k\left\|x_{0}\right\| \exp \left(-\beta\left(t-t_{0}\right)\right)+r, \forall t \geq t_{0}$ (Laskhmikantam et al. [1990]).

\section{BACKSTEPPING CONTROL DESIGN}

This part deals with the speed and flux control by means of backstepping control. This nonlinear control technique can be applied efficiently to linearize a nonlinear system with the existence of uncertainties, it is usually incorporated with the nonlinear damping to enhance robustness (Chen [1996], Polycarpou et al. [1993]).

In this work in order to compensate the rotor resistance variations and the load disturbance the backstepping technique is used. The idea of backstepping design is to select recursively some appropriate functions of state variables as virtual control inputs for lower dimension subsystems of the overall system. At each step of the backstepping a new virtual control input is designed. When the procedure terminates, the actual control input results which achieves the original design objective by virtue of a final Lyapunov function, which is formed by summing up the Lyapunov functions associated with each individual design step.

\subsection{Step1: Flux control}

The objective is to steer the flux $\phi_{d r}$ to a desired reference $\phi_{d r}^{*}$, let $e_{\phi}=\phi_{d r}-\phi_{d r}^{*}$ be the flux tracking error. The dynamic of $e_{\phi}$ is:

$$
\dot{e}_{\phi}=\frac{L_{m}}{\tau_{r}} i_{d s}-\frac{1}{\tau_{r}} \phi_{d r}+h_{3}(x)-\dot{\phi}_{d r}^{*}
$$

A Lyapunov function is defined as:

$$
V_{\phi}=\frac{1}{2} e_{\phi}^{2}
$$

By deriving (6) we obtain:

$$
\dot{V}_{\phi}=e_{\phi} \dot{e}_{\phi}=e_{\phi}\left(\frac{L_{m}}{\tau_{r}} i_{d s}-\frac{1}{\tau_{r}} \phi_{d r}+h_{3}(x)-\dot{\phi}_{d r}^{*}\right)
$$

To make $\dot{V}_{\phi}$ negative definite, $i_{d s}$ is chosen as virtual element of control for stabilizing the flux, its desired value $i_{d s}^{*}$ is defined as:

$$
i_{d s}^{*}=\frac{\tau_{r}}{L_{m}}\left(-k_{\phi} e_{\phi}-k_{1} \tanh \left(\frac{k_{1} h}{\varepsilon_{1}} e_{\phi}\right)+\frac{\phi_{d r}}{\tau_{r}}+\dot{\phi}_{d r}^{*}\right)
$$

where $h=0.2785 . k_{1}, k_{\phi}$ and $\varepsilon_{1}$ are positive design parameters.

By setting $i_{d s}=i_{d s}^{*}$ in (7) we get :

$$
\dot{V}_{\phi}=-k_{\phi} e_{\phi}^{2}-k_{1} \tanh \left(\frac{k_{1} h}{\varepsilon_{1}} e_{\phi}\right) e_{\phi}+h_{3} e_{\phi}
$$

for $k_{1}>\left|h_{3}\right|_{\max }$ we get:

$$
\dot{V}_{\phi} \leq-k_{\phi} e_{\phi}^{2}-k_{1} \tanh \left(\frac{k_{1} h}{\varepsilon_{1}} e_{\phi}\right) e_{\phi}+k_{1}\left|e_{\phi}\right|
$$

with:

$$
\left|e_{\phi}\right|=e_{\phi} \text { signe }_{\phi}
$$

The derivative of the Lyapunov function (10) becomes:

$$
\dot{V}_{\phi} \leq-k_{\phi} e_{\phi}^{2}-k_{1} \tanh \left(\frac{k_{1} h}{\varepsilon_{1}} e_{\phi}\right) e_{\phi}+k_{1} e_{\phi} \operatorname{signe}_{\phi}
$$


we have (see Polycarpou et al. [1993]):

$$
0 \leq k_{1} e_{\phi} \text { signe }_{\phi}-k_{1} \tanh \left(\frac{k_{1} h}{\varepsilon_{1}} e_{\phi}\right) e_{\phi} \leq \varepsilon_{1}
$$

The derivative of the Lyapunov function (12) becomes:

$$
\dot{V}_{\phi} \leq-k_{\phi} e_{\phi}^{2}+\varepsilon_{1}
$$

This implies that the variable $e_{\phi}$ converges to a ball whose radius can be reduced by making small the tuning parameter $\varepsilon_{1}$.

\subsection{Step2: Speed control}

The objective is to steer the speed $\Omega$ to the desired reference $\Omega^{*}$, let $e_{\Omega}=\Omega-\Omega^{*}$ be the speed tracking error. The error dynamic of the speed is:

$$
\dot{e}_{\Omega}=\frac{P L_{m}}{L_{r} J} i_{q s} \phi_{d r}-\frac{f}{J} \Omega-\frac{T}{J}-\dot{\Omega}^{*}
$$

A Lyapunov function is defined as:

$$
V_{\Omega}=\frac{1}{2} e_{\Omega}^{2}
$$

By deriving (16) we obtain:

$$
\dot{V}_{\Omega}=e_{\Omega} \dot{e}_{\Omega}=e_{\Omega}\left(\frac{P L_{m}}{L_{r} J} i_{q s} \phi_{d r}-\frac{f}{J} \Omega-\frac{T}{J}-\dot{\Omega}^{*}\right)
$$

$i_{q s}$ is chosen as virtual element of control for stabilizing the speed, its desired value $i_{q s}^{*}$ is defined as:

$i_{q s}^{*}=\frac{J L_{r}}{L_{m} P \phi_{d r}}\left(-k_{\Omega} e_{\Omega}-k_{2} \tanh \frac{k_{2} h}{\varepsilon_{2}} e_{\Omega}+\frac{f}{J} \Omega+\dot{\Omega}^{*}\right), \phi_{d r} \neq 0$

where $h=0.2785 . k_{2}$ and $k_{\Omega}$ and $\varepsilon_{2}$ are positive design parameters.

By setting $i_{q s}=i_{q s}^{*}$ in (17) we get:

$$
\dot{V}_{\Omega}=e_{\Omega}\left(-k_{\Omega} e_{\Omega}-k_{2} \tanh \frac{k_{2} h}{\varepsilon_{2}} e_{\Omega}-\frac{T}{J}\right)
$$

For $k_{2}>\left|\frac{T}{J}\right|_{\max }$ we obtain:

$\dot{V}_{\Omega} \leq-k_{\Omega} e_{\Omega}^{2}-k_{2} \tanh \left(\frac{k_{2} h}{\varepsilon_{2}} e_{\Omega}\right) e_{\Omega}+k_{2}\left|e_{\Omega}\right| \leq-k_{\Omega} e_{\Omega}^{2}+\varepsilon_{2}$

This implies that the variable $e_{\Omega}$ converges to a ball whose radius can be reduced by making small the tuning parameter $\varepsilon_{2}$.

\subsection{Step3: Currents control}

The objective is to steer the currents $i_{d s}$ and $i_{q s}$ to their desired references $i_{d s}^{*}$ and $i_{q s}^{*}$, respectively. Let $e_{d}=i_{d s}-$ $i_{d s}^{*}$ and $e_{q}=i_{q s}-i_{q s}^{*}$ be the tracking errors of the currents, then the dynamic of the tracking errors are:

$$
\begin{aligned}
& \dot{e}_{d}=-a i_{d s}+\omega_{s} i_{q s}+\frac{L_{m}}{\sigma L_{s} L_{r} \tau_{r}} \phi_{d r}+\frac{V_{d s}}{\sigma L_{s}}+h_{1}(x)-\frac{d i_{d s}^{*}}{d t} \\
& \dot{e}_{q}=-a i_{q s}-\omega_{s} i_{d s}-\frac{L_{m}}{\sigma L_{s} L_{r}} P \Omega \phi_{d r}+\frac{V_{q s}}{\sigma L_{s}}+h_{2}(x)-\frac{d i_{q s}^{*}}{d t} \\
& \dot{e_{\phi}}=\frac{L_{m}}{\tau_{r}} e_{d}+\frac{L_{m}}{\tau_{r}} i_{d s}^{*}-\frac{1}{\tau_{r}} \phi_{d r}+h_{3}(x)-\dot{\phi}_{d r}^{*} \\
& \dot{e}_{\Omega}=\frac{P L_{m}}{L_{r} J} e_{q} \phi_{d r}+\frac{P L_{m}}{L_{r} J} i_{q s}^{*} \phi_{d r}-\frac{f}{J} \Omega-\frac{T}{J}-\dot{\Omega}^{*}
\end{aligned}
$$

with:

$$
\begin{aligned}
i_{d s}^{*}= & \frac{\tau_{r}}{L_{m}}\left(-k_{\phi} e_{\phi}-k_{1} \tanh \left(\frac{k_{1} h}{\varepsilon_{1}} e_{\phi}\right)+\frac{\phi_{d r}}{\tau_{r}}+\dot{\phi}_{d r}^{*}\right) \\
i_{q s}^{*}= & \frac{J L_{r}}{L_{m} P \phi_{d r}}\left(-k_{\Omega} e_{\Omega}-k_{2} \tanh \frac{k_{2} h}{\varepsilon_{2}} e_{\Omega}+\frac{f}{J} \Omega+\dot{\Omega}^{*}\right) \\
\frac{d i_{d s}^{*}}{d t}= & \frac{\tau_{r}}{L_{m}} F_{1}\left(e_{\phi}\right)\left(\frac{L_{m}}{\tau_{r}} i_{d s}-\frac{\phi_{d r}}{\tau_{r}}+h_{3}(x)\right) \\
& -\frac{\tau_{r}}{L_{m}}\left(F_{1}\left(e_{\phi}\right)-\frac{1}{\tau_{r}}\right) \dot{\phi}_{d r}^{*}+\frac{\tau_{r}}{L_{m}} \ddot{\phi}_{d r}^{*} \\
\frac{d i_{q s}^{*}}{d t}= & \frac{J L_{r}}{L_{m} P \phi_{d r}} F_{2}\left(e_{\Omega}\right)\left(\frac{P L_{m}}{L_{r} J} i_{q s} \phi_{d r}-\frac{f}{J} \Omega\right) \\
& +F_{3}\left(e_{\Omega}, \Omega, \phi_{d r}\right)+\frac{J L_{r}}{L_{m} P \phi_{d r}}\left(\frac{f}{J}-F_{2}\left(e_{\Omega}\right)\right) \dot{\Omega}^{*} \\
& +\frac{J L_{r}}{L_{m} P \phi_{d r}} \ddot{\Omega}^{*}-\frac{L_{r} F_{2}\left(e_{\Omega}\right)}{P L_{m} \phi_{d r}} T+F_{4} h_{3}(x)
\end{aligned}
$$

where:

$$
\begin{gathered}
F_{1}\left(e_{\phi}\right)=-k_{\phi}-\frac{k_{1}^{2} h}{\varepsilon_{1}}\left(1-\tanh \left(\frac{k_{1} h}{\varepsilon_{1}} e_{\phi}\right)^{2}\right)+\frac{1}{\tau_{r}} \\
F_{2}\left(e_{\Omega}\right)=-k_{\Omega}-\frac{k_{2}^{2} h}{\varepsilon_{2}}\left(1-\tanh \left(\frac{k_{2} h}{\varepsilon_{2}} e_{\Omega}\right)^{2}\right)+\frac{f}{J} \\
F_{3}\left(e_{\Omega}, \Omega, \phi_{d r}\right)=\left(\frac{L_{m}}{\tau_{r}} i_{d s}-\frac{\phi_{d r}}{\tau_{r}}\right) F_{4}\left(e_{\Omega}, \Omega, \phi_{d r}\right) \\
F_{4}\left(e_{\Omega}, \Omega, \phi_{d r}\right)=\frac{J L_{r}}{P L_{m} \phi_{d r}^{2}}\left(k_{\Omega} e_{\Omega}+k_{2} \tanh \left(\frac{k_{2} h}{\varepsilon_{2}} e_{\Omega}\right)\right) \\
+\frac{J L_{r}}{P L_{m} \phi_{d r}^{2}}\left(-\frac{f}{J} \Omega-\dot{\Omega}^{*}\right)
\end{gathered}
$$

By substituting $i_{d s}^{*}, i_{q s}^{*}, \frac{d i_{d s}^{*}}{d t}$ and $\frac{d i_{q s}^{*}}{d t}$ by their expressions, the system of the tracking errors (21) becomes:

$$
\begin{aligned}
\dot{e}_{d}= & -a i_{d s}+\omega_{s} i_{q s}+\frac{L_{m}}{\sigma L_{s} L_{r} \tau_{r}} \phi_{d r}+\frac{V_{d s}}{\sigma L_{s}}+h_{1}(x) \\
& -\frac{\tau_{r}}{L_{m}} F_{1}\left(e_{\phi}\right)\left(\frac{L_{m}}{\tau_{r}} i_{d s}-\frac{\phi_{d r}}{\tau_{r}}+h_{3}(x)\right) \\
& +\frac{\tau_{r}}{L_{m}}\left(F_{1}\left(e_{\phi}\right)-\frac{1}{\tau_{r}}\right) \dot{\phi}_{d r}^{*}-\frac{\tau_{r}}{L_{m}} \ddot{\phi}_{d r}^{*} \\
\dot{e}_{q}= & -a i_{q s}-\omega_{s} i_{d s}-\frac{L_{m}}{\sigma L_{s} L_{r}} P \Omega \phi_{d r}+\frac{V_{q s}}{\sigma L_{s}}+h_{2}(x) \\
& -\frac{J L_{r}}{L_{m} P \phi_{d r}} F_{2}\left(e_{\Omega}\right)\left(\frac{P L_{m}}{L_{r} J} i_{q s} \phi_{d r}-\frac{f}{J} \Omega\right) \\
& -F_{3}\left(e_{\Omega}, \Omega, \phi_{d r}\right)-\frac{J L_{r}}{L_{m} P \phi_{d r}}\left(\frac{f}{J}-F_{2}\left(e_{\Omega}\right)\right) \dot{\Omega}^{*} \\
& -\frac{J L_{r}}{L_{m} P \phi_{d r}} \ddot{\Omega}^{*}-\frac{L_{r} F_{2}\left(e_{\Omega}\right)}{P L_{m} \phi_{d r}} T-F_{4} h_{3}(x) \\
\dot{e}_{\phi}= & -k_{\phi} e_{\phi}-k_{1} \tanh \left(\frac{k_{1} h}{\varepsilon_{1}} e_{\phi}\right)+\frac{L_{m}}{\tau_{r}} e_{d}+h_{3}(x) \\
\dot{e}_{\Omega}= & \frac{P L_{m}}{L_{r} J} e_{q} \phi_{d r}-k_{\Omega} e_{\Omega}-k_{2} \tanh \left(\frac{k_{2} h}{\varepsilon_{2}} e_{\Omega}\right)-\frac{T}{J}
\end{aligned}
$$

The actual control inputs are chosen as follows: 


$$
\begin{aligned}
V_{d s}= & \sigma L_{s}\left(-k_{d} e_{d}-k_{3} \tanh \left(\frac{k_{3} h}{\varepsilon_{3}} e_{d}\right)+a i_{d s}-\frac{L_{m}}{\tau_{r}} e_{\phi}\right. \\
& -\omega_{s} i_{q s}-\frac{L_{m}}{\sigma L_{s} L_{r} \tau_{r}} \phi_{d r}+\frac{\tau_{r}}{L_{m}} F_{1}\left(\frac{L_{m}}{\tau_{r}} i_{d s}-\frac{\phi_{d r}}{\tau_{r}}\right) \\
& \left.-\frac{\tau_{r}}{L_{m}}\left(F_{1}-\frac{1}{\tau_{r}}\right) \dot{\phi}_{d r}^{*}+\frac{\tau_{r}}{L_{m}} \ddot{\phi}_{d r}^{*}\right) \\
V_{q s}= & \sigma L_{s}\left(-k_{q} e_{q}-k_{4} \tanh \left(\frac{k_{4} h}{\varepsilon_{4}} e_{q}\right)+a i_{q s}+\omega_{s} i_{d s}\right. \\
& +\frac{L_{m}}{\sigma L_{s} L_{r}} P \Omega \phi_{d r}-\frac{P L_{m}}{J L_{r}} e_{\Omega} \phi_{d r}+\frac{J L_{r}}{L_{m} P \phi_{d r}} F_{2}\left(e_{\Omega}\right) \\
& \left(\frac{P L_{m}}{L_{r} J} i_{q s} \phi_{d r}-\frac{f}{J} \Omega\right)+F_{3}\left(e_{\Omega}, \Omega, \phi_{d r}\right) \\
& \left.+\frac{J L_{r}}{L_{m} P \phi_{d r}}\left(\frac{f}{J}-F_{2}\left(e_{\Omega}\right)\right) \dot{\Omega}^{*}+\frac{J L_{r}}{L_{m} P \phi_{d r}} \ddot{\Omega}^{*}\right)
\end{aligned}
$$

Proposition 1. Consider the system (3) and the control inputs (24) and (25) where : $k_{d}, k_{q}, k_{3}, k_{4}$ are positive design parameters. $\varepsilon_{1}, \varepsilon_{2}, \varepsilon_{3}$ and $\varepsilon_{4}$ are positive and arbitrary small parameters. Then, if $k_{3}>\left|h_{1}(x)-\frac{\tau_{r}}{L_{m}} F_{1} h_{3}(x)\right|_{\max }$ and $k_{4}>\left|h_{2}(x)-F_{4} h_{3}(x)+\frac{L_{r} F_{2}\left(e_{\Omega}\right)}{P L_{m} \phi_{d r}} T\right|_{\text {max }}$, the error variables $e_{\phi}, e_{\Omega}, e_{d}$ and $e_{q}$ are globally uniformly exponentially practically stable.

Proof. By substituting the control laws (24) and (25) in the error system (23) we get:

$$
\begin{aligned}
\dot{e_{d}}= & -k_{d} e_{d}-k_{3} \tanh \left(\frac{k_{3} h}{\varepsilon_{3}} e_{d}\right)-\frac{L_{m}}{\tau_{r}} e_{\phi}+h_{1}(x) \\
& -\frac{\tau_{r}}{L_{m}} F_{1} h_{3}(x) \\
\dot{e_{q}}= & -k_{q} e_{q}-k_{4} \tanh \left(\frac{k_{4} h}{\varepsilon_{4}} e_{q}\right)-\frac{P L_{m}}{J L_{r}} e_{\Omega} \phi_{d r}+h_{2}(x) \\
& -F_{4} h_{3}(x)+\frac{L_{r} F_{2}\left(e_{\Omega}\right)}{P L_{m} \phi_{d r}} T \\
\dot{e}_{\phi}= & -k_{\phi} e_{\phi}-k_{1} \tanh \left(\frac{k_{1} h}{\varepsilon_{1}} e_{\phi}\right)+\frac{L_{m}}{\tau_{r}} e_{d}+h_{3}(x) \\
\dot{e}_{\Omega}= & \frac{P L_{m}}{L_{r} J} e_{q} \phi_{d r}-k_{\Omega} e_{\Omega}-k_{2} \tanh \left(\frac{k_{2} h}{\varepsilon_{2}} e_{\Omega}\right)-\frac{T}{J}
\end{aligned}
$$

Consider the following Lyapunov function:

$$
V=\frac{1}{2}\left(e_{d}^{2}+e_{q}^{2}+e_{\phi}^{2}+e_{\Omega}^{2}\right)
$$

The derivative of $V$ with respect to time is:

$$
\begin{aligned}
\dot{V}= & e_{d}\left(-k_{d} e_{d}-k_{3} \tanh \left(\frac{k_{3} h}{\varepsilon_{3}} e_{d}\right)-\frac{L_{m}}{\tau_{r}} e_{\phi}+h_{1}(x)\right. \\
& \left.-\frac{\tau_{r}}{L_{m}} F_{1} h_{3}(x)\right) \\
& +e_{q}\left(-k_{q} e_{q}-k_{4} \tanh \left(\frac{k_{4} h}{\varepsilon_{4}} e_{q}\right)-\frac{P L_{m}}{J L_{r}} e_{\Omega} \phi_{d r}+h_{2}(x)\right. \\
& \left.-F_{4} h_{3}(x)+\frac{L_{r} F_{2}\left(e_{\Omega}\right)}{P L_{m} \phi_{d r}} T\right) \\
& +e_{\phi}\left(-k_{\phi} e_{\phi}-k_{1} \tanh \left(\frac{k_{1} h}{\varepsilon_{1}} e_{\phi}\right)+\frac{L_{m}}{\tau_{r}} e_{d}+h_{3}(x)\right) \\
+ & e_{\Omega}\left(\frac{P L_{m}}{L_{r} J} e_{q} \phi_{d r}-k_{\Omega} e_{\Omega}-k_{2} \tanh \left(\frac{k_{2} h}{\varepsilon_{2}} e_{\Omega}\right)-\frac{T}{J}\right)
\end{aligned}
$$

From the step 1 and 2 we have $k_{1}>\left|h_{3}\right|_{\max }$ and $k_{2}>$ $\left|\frac{T}{J}\right|_{\text {max }}$, then the derivative of the Lyapunov function (28) becomes:

$$
\begin{aligned}
\dot{V} \leq & -k_{\phi} e_{\phi}^{2}-k_{\Omega} e_{\Omega}^{2}+\varepsilon_{1}+\varepsilon_{2}-k_{d} e_{d}^{2}-k_{q} e_{q}^{2} \\
& -k_{3} \tanh \left(\frac{k_{3} h}{\varepsilon_{3}} e_{d}\right) e_{d}-k_{4} \tanh \left(\frac{k_{4} h}{\varepsilon_{4}} e_{q}\right) e_{q} \\
& +\left(h_{2}(x)-F_{4} h_{3}(x)+\frac{L_{r} F_{2}\left(e_{\Omega}\right)}{P L_{m} \phi_{d r}} T\right) e_{q} \\
& +\left(h_{1}(x)-\frac{\tau_{r}}{L_{m}} F_{1}\left(e_{\phi}\right) h_{3}(x)\right) e_{d}
\end{aligned}
$$

For

$$
k_{3}>\left|h_{1}(x)-\frac{\tau_{r}}{L_{m}} F_{1} h_{3}(x)\right|_{\max }
$$

and

we get:

$$
k_{4}>\left|h_{2}(x)-F_{4} h_{3}(x)+\frac{L_{r} F_{2}\left(e_{\Omega}\right)}{P L_{m} \phi_{d r}} T\right|_{\max }
$$

$$
\dot{V} \leq-k_{\phi} e_{\phi}^{2}-k_{\Omega} e_{\Omega}^{2}-k_{d} e_{d}^{2}-k_{q} e_{q}^{2}+\varepsilon_{1}+\varepsilon_{2}+\varepsilon_{3}+\varepsilon_{4}
$$

This implies that the error variables $e_{\phi}, e_{\Omega}, e_{d}$ and $e_{q}$ converge to a ball whose radius can be reduced by making small the tuning parameters $\varepsilon_{1}, \varepsilon_{2}, \varepsilon_{3}$ and $\varepsilon_{4}$. This means that the error variables are globally uniformly exponentially practically stable (see the definition 1).

In order to implement the control laws without flux and speed sensors, a second order sliding mode observer is used to estimate the speed $\Omega$ and the flux $\phi_{d r}$.

\section{SECOND ORDER SLIDING MODE OBSERVER DESIGN}

The IM model in $(\alpha-\beta)$ reference frame is given by:

$$
\begin{aligned}
\dot{i}_{\alpha s} & =-a i_{\alpha s}+\frac{L_{m}}{\sigma L_{s} L_{r} \tau_{r}} \phi_{\alpha r}+\frac{L_{m}}{\sigma L_{s} L_{r}} P \Omega \phi_{\beta r}+\frac{V_{\alpha s}}{\sigma L_{s}} \\
\dot{i}_{\beta s} & =-a i_{\beta s}-\frac{L_{m}}{\sigma L_{s} L_{r}} P \Omega \phi_{\alpha r}+\frac{V_{m s}}{\sigma L_{s} L_{r} \tau_{r}} \phi_{\beta r}+\frac{1}{\sigma L_{s}} \\
\dot{\phi}_{\alpha r} & =-P \Omega \phi_{\beta r}+\frac{L_{m}}{\tau_{r}} i_{\alpha s}-\frac{1}{\tau_{r}} \phi_{\alpha r} \\
\dot{\phi}_{\beta r} & =P \Omega \phi_{\alpha r}+\frac{L_{m}}{\tau_{r}} i_{\beta s}-\frac{1}{\tau_{r}} \phi_{\beta r} \\
\dot{\Omega} & =\frac{P L_{m}}{L_{r} J}\left(i_{\beta s} \phi_{\alpha r}-i_{\alpha s} \phi_{\beta r}\right)-\frac{f}{J} \Omega-\frac{T}{J}
\end{aligned}
$$

with $V_{\alpha s}, V_{\beta s}$ are stator voltage components. $\phi_{\alpha r}, \phi_{\beta r}$ are the rotor flux components. $\Omega$ is the mechanical speed. $T$ is the load torque. $i_{\alpha s}, i_{\beta s}$ are stator current components. The currents $i_{\alpha s}, i_{\beta s}$ are assumed to be measured. By applying the following change of variable:

$$
\begin{aligned}
z_{1} & =i_{\alpha s} \\
z_{2} & =i_{\beta s} \\
z_{3} & =\frac{L_{m}}{\sigma L_{s} L_{r} \tau_{r}} \phi_{\alpha r}+\frac{L_{m}}{\sigma L_{s} L_{r}} P \Omega \phi_{\beta r} \\
z_{4} & =-\frac{L_{m}}{\sigma L_{s} L_{r}} P \Omega \phi_{\alpha r}+\frac{L_{m}}{\sigma L_{s} L_{r} \tau_{r}} \phi_{\beta r} \\
z_{5} & =\dot{z}_{3} \\
z_{6} & =\dot{z}_{4}
\end{aligned}
$$

the system (31) becomes as follows: 


$$
\begin{aligned}
& \dot{z}_{1}=-a z_{1}+z_{3}+\frac{V_{\alpha s}}{\sigma L_{s}} \\
& \dot{z}_{2}=-a z_{2}+z_{4}+\frac{V_{\beta s}}{\sigma L_{s}} \\
& \dot{z}_{3}=z_{5} \\
& \dot{z}_{4}=z_{6} \\
& \dot{z}_{5}=z_{7} \\
& \dot{z}_{6}=z_{8}
\end{aligned}
$$

A second order sliding mode observer is defined as (Levant [1998], Floquet et al. [2007]):

$$
\begin{aligned}
& \dot{\tilde{z}}_{1}=-a z_{1}+\tilde{z}_{3}+\lambda_{1}\left|z_{1}-\hat{z}_{1}\right|^{0.5} \operatorname{sign}\left(z_{1}-\hat{z}_{1}\right)+\frac{V_{\alpha s}}{\sigma L_{s}} \\
& \dot{\tilde{z}}_{3}=\alpha_{1} \operatorname{sign}\left(z_{1}-\hat{z}_{1}\right) \\
& \dot{\tilde{z}}_{2}=-a z_{2}+\tilde{z}_{4}+\lambda_{2}\left|z_{2}-\hat{z}_{2}\right|^{0.5} \operatorname{sign}\left(z_{2}-\hat{z}_{2}\right)+\frac{V_{\beta s}}{\sigma L_{s}} \\
& \dot{\tilde{z}}_{4}=\alpha_{2} \operatorname{sign}\left(z_{2}-\hat{z}_{2}\right) \\
& \dot{\hat{z}}_{3}=E_{1} E_{2}\left(\tilde{z}_{5}+\lambda_{3}\left|\tilde{z}_{3}-\hat{z}_{3}\right|^{0.5} \operatorname{sign}\left(\tilde{z}_{3}-\hat{z}_{3}\right)\right) \\
& \dot{\tilde{z}}_{5}=E_{1} E_{2} \alpha_{3} \operatorname{sign}\left(\tilde{z}_{3}-\hat{z}_{3}\right) \\
& \dot{\tilde{z}}_{4}=E_{1} E_{2}\left(\tilde{z}_{6}+\lambda_{4}\left|\tilde{z}_{4}-\hat{z}_{4}\right|^{0.5} \operatorname{sign}\left(\tilde{z}_{4}-\hat{z}_{4}\right)\right) \\
& \dot{\tilde{z}}_{6}=E_{1} E_{2} \alpha_{4} \operatorname{sign}\left(\tilde{z}_{4}-\hat{z}_{4}\right) \\
& \dot{\tilde{z}}_{5}=E_{1} E_{2} E_{3} E_{4}\left(\tilde{z}_{7}+\lambda_{5}\left|\tilde{z}_{5}-\hat{z}_{5}\right|^{0.5} \operatorname{sign}\left(\tilde{z}_{5}-\hat{z}_{5}\right)\right) \\
& \dot{\tilde{z}}_{7}=E_{1} E_{2} E_{3} E_{4} \alpha 5 \operatorname{sign}\left(\tilde{z}_{5}-\hat{z}_{5}\right) \\
& \dot{\tilde{z}}_{6}=E_{1} E_{2} E_{3} E_{4}\left(\tilde{z}_{8}+\lambda_{6}\left|\tilde{z}_{6}-\hat{z}_{6}\right|^{0.5} \operatorname{sign}\left(\tilde{z}_{6}-\hat{z}_{6}\right)\right) \\
& \dot{\tilde{z}}_{8}=E_{1} E_{2} E_{3} E_{4} \alpha_{6} \operatorname{sign}\left(\tilde{z}_{6}-\hat{z}_{6}\right)
\end{aligned}
$$

where $E_{i}=1$ if $\tilde{z}_{i}-\hat{z}_{i}=0$ else $E_{i}=0$ for $\mathrm{i}=1, \ldots, \mathrm{n}$. with $\tilde{z}_{1}=z_{1}, \tilde{z}_{2}=z_{2}$. For a suitable choice of the parameters $\lambda_{i}$ and $\alpha_{i}: \alpha_{1}>z_{5 \max }, \lambda_{1}>\left(\alpha_{1}+z_{5 \max }\right) \sqrt{\frac{2}{\alpha_{1}-z_{5 \max }}}$, $\alpha_{2}>z_{6 \max }, \lambda_{2}>\left(\alpha_{2}+z_{6 \max }\right) \sqrt{\frac{2}{\alpha_{2}-z_{6 \max }}}$...etc (for proof see Levant [1998], Floquet et al. [2007]), the observation errors $\left(\tilde{z}_{i}-\hat{z}_{i}\right)$ tend to zero in finite time, then the speed and the flux are estimated as follows:

From equations (32) we have:

$$
\begin{aligned}
& z_{3}=b \phi_{\alpha r}+c \Omega \phi_{\beta r} \\
& z_{4}=-c \Omega \phi_{\alpha r}+b \phi_{\beta r}
\end{aligned}
$$

where: $b=\frac{L_{m}}{\sigma L_{s} L_{r} \tau_{r}}, c=\frac{L_{m}}{\sigma L_{s} L_{r}} P$

By solving the above equations we get:

$$
\begin{aligned}
& \phi_{\alpha r}=\frac{b z_{3}-c \Omega z_{4}}{b^{2}+c^{2} \Omega^{2}} \\
& \phi_{\beta r}=\frac{c \Omega z_{3}+b z_{4}}{b^{2}+c^{2} \Omega^{2}}
\end{aligned}
$$

By substituting $z_{3}, z_{4}$ and $\Omega$ by their estimates $\hat{z}_{3}, \hat{z}_{4}$ and $\hat{\Omega}$ we obtain the flux estimates as follows:

$$
\begin{gathered}
\hat{\phi}_{\alpha r}=\frac{b \hat{z}_{3}-c \hat{\Omega} \hat{z}_{4}}{b^{2}+c^{2} \hat{\Omega}^{2}} \\
\hat{\phi}_{\beta r}=\frac{c \hat{\Omega} \hat{z}_{3}+b \hat{z}_{4}}{b^{2}+c^{2} \hat{\Omega}^{2}}
\end{gathered}
$$

By deriving the equations (35) we get:

$$
\begin{aligned}
& z_{5}=\dot{z}_{3}=-\frac{1}{\tau_{r}} z_{3}-P \Omega z_{4}+b \frac{L_{m}}{\tau_{r}} i_{\alpha s}+c \frac{L_{m}}{\tau_{r}} \Omega i_{\beta s}+c \phi_{\beta r} \dot{\Omega} \\
& z_{6}=\dot{z}_{4}=-\frac{1}{\tau_{r}} z_{4}+P \Omega z_{3}+b \frac{L_{m}}{\tau_{r}} i_{\beta s}-c \frac{L_{m}}{\tau_{r}} \Omega i_{\alpha s}-c \phi_{\alpha r} \dot{\Omega}
\end{aligned}
$$

Finally, by neglecting the speed variation $\dot{\Omega}$, the estimate of the speed is obtained from the above equations as follows:

$$
\hat{\Omega}=\frac{\hat{z}_{5}+\frac{1}{\tau_{r}} \hat{z}_{3}-b \frac{L_{m}}{\tau_{r}} i_{\alpha s}}{c \frac{L_{m}}{\tau_{r}} i_{\beta s}-P \hat{z}_{4}}
$$

\section{SIMULATION RESULTS}

Numerical simulations have been performed to validate the proposed control scheme. The IM parameters are given in the appendix. The controller parameters are chosen as follows: $k_{\Omega}=0.5, k_{\phi}=10, k_{1}=10, k_{2}=300, k_{3}=100$, $k_{4}=100, k_{d}=700$ and $k_{q}=500$. The speed and flux references are fixed at $\Omega_{*}=100 \mathrm{rad} / \mathrm{s}$ and $\phi_{d r}^{*}=0.9 \mathrm{~Wb}$, respectively, also a load disturbance $T=3 N . m$ is applied. Figure 1 shows the responses of the IM without parameters variations (un-faulty mode), we see that the speed and the flux trajectories converge to their desired references, also the estimated flux and speed converge to their actual values. Figure 2 and 3 show the responses of the IM with rotor resistance variations of $+50 \% R_{r}$ and $+100 \% R_{r}$, respectively. In each case, the controller rejects the rotor resistance variations.

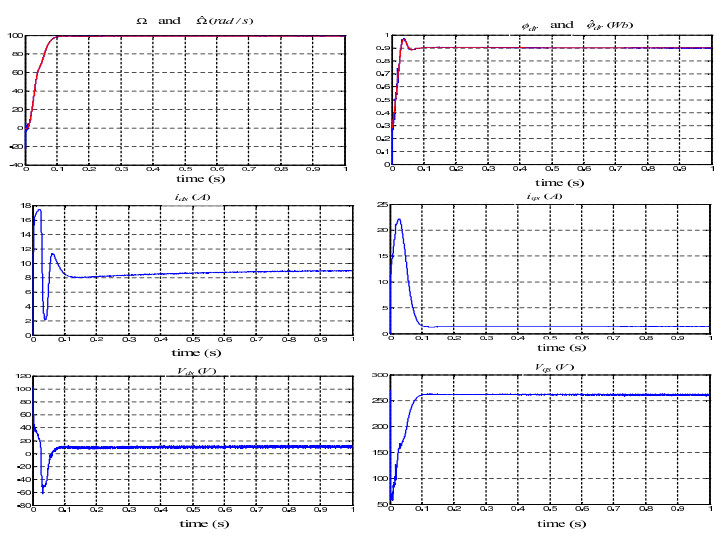

Fig. 1. Responses of the IM in un-faulty mode
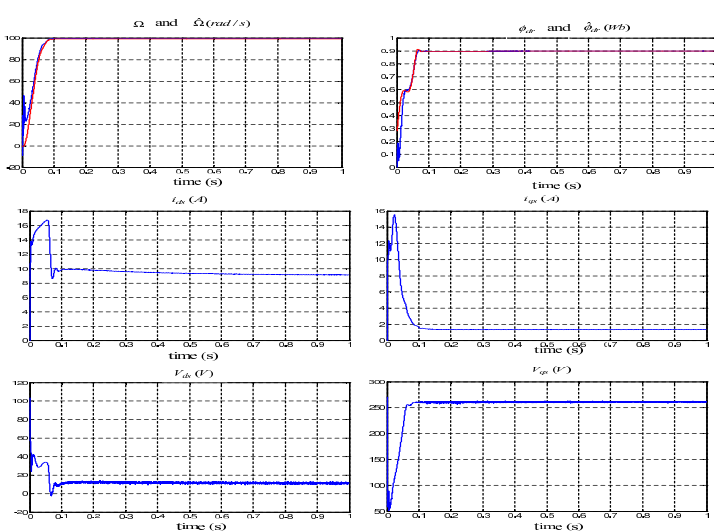

Fig. 2. Responses of the IM with rotor resistance variation of $+50 \% \mathrm{Rr}$ 

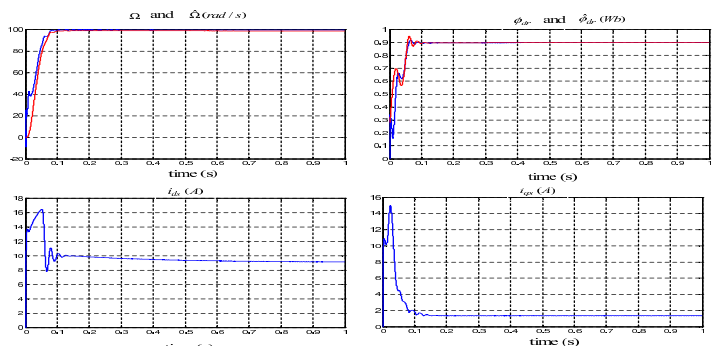

time (s)
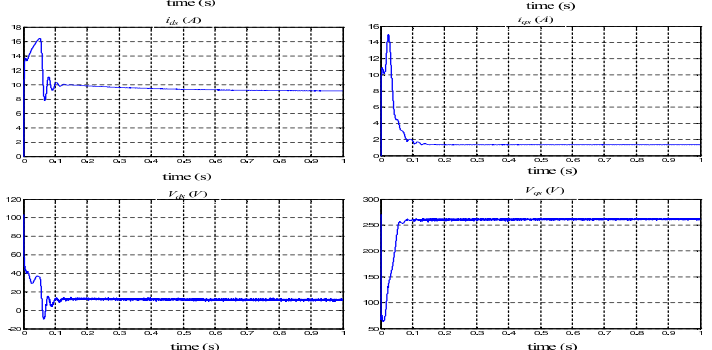

Fig. 3. Responses of the IM with rotor resistance variation of $+100 \% \mathrm{Rr}$

\section{CONCLUSION}

In this paper a sensorless fault tolerant controller for IM has been presented. First, a field oriented controller based on backstepping strategy is designed to steer the flux and the speed to their desired references in presence of rotor resistance variations and load torque disturbance. Second, to achieve the sensorless fault tolerant control, a second order sliding mode observer is used to estimate the speed and the flux from the stator currents measurements. The simulation results show the robustness of the proposed control scheme. Certain points remain to be studied. The stability analysis of the closed loop system (observer+controller) and the experimental validation of the proposed control scheme will be included in the future work.

\section{REFERENCES}

M. Blanke, M. Kinnaert, J. Lunze, M. Staroswiecki, Diagnostic and fault tolerant control, Springer-Verlag, 2003.

M. Blanke, M. Staroswiecki and N. Wu, Concepts and methods in fault-tolerant control, Proceedings of the American Control Conference, Arlington, 2606-2020, 2001.

C. Bonivento, A. Isidori, L. Marconi and A. Paoli, Implicit fault tolerant control: application to induction motors. Automatica, 355-371, 2004.

C. Canudas de Wit, Commande des moteurs asynchrones 2, Optimisation, discrétisation et observateurs, Edition Hermes Science, Paris, 2000.

C. Chen, Backstepping control design and its applications to vehicle lateral control in automated highway systems, Dissertation, University of California at Berkeley, 1996.

D. Diallo, M. E. H. Benbouzid, A. Makouf: A fault tolerant control architecture for induction motor drives in automotive applications, IEEE Trans. Veh. Technol., 1847-1855, 2004.

N. Djeghali, M. Ghanes, S. Djennoune, J. P. Barbot and M. Tadjine, Fault tolerant control for induction motors using sliding mode observer, The 11th International Workshop On Variable Structure Systems, Mexico City, 190-196, 2010.

C. Edwards, S. K. Spurgeon and R. J. Patton, Sliding mode observers for fault detection and isolation, Automatica, 36, 541-553, 2000.
A. Fekih, Effective fault tolerant control design for nonlinear systems: application to a class of motor control system, IET Control Theory Appl., 762-772, 2008.

T. Floquet and J.P. Barbot, Super twisting algorithm based step by step sliding mode observers for nonlinear systems with unknown inputs, International Journal of Systems Science, 2007.

M. Ghanes and G. Zheng, On sensorless induction motor drives: Sliding mode observer and output feedback controller, IEEE Trans.On Industrial Electronics, 34043413, 2009.

M. Ghanes, J. P. Barbot, J. D. Leon and A. Glumineau, A robust sensorless output feedback controller of the induction drives: New design and experimental validation, Int. J. of Control, 2010.

J. Holtz, Sensorless control of induction machines with or without signal injection, IEEE Trans. Ind. Electron., 730, 2006.

V. Laskhmikantham, S. Leila and A. A. Martynyuk, Practical stability of nonlinear systems, Word Scientific, 1990.

A. Levant, Robust exact differentiation via sliding mode technique, Automatica, 379-384, 1998.

S. Moreau, J. C. Trigeassou, G. Chaampenois and J. P. Gaubert, Diagnostic of induction machines: A procedure for electrical fault detection and localisation, SDEMPED, 1999.

W. Perruquetti and J. P. Barbot, Sliding mode control in engineering, Control Engineering Series, 2002.

M. Polycarpou and P. Ioannou, A robust Adaptive Nonlinear Control Design, Proceedings of American Control Conference, 1365-1369, 1993.

S. Solvar, V. Le, M. Ghanes, J. P. Barbot and G. Santomenna, Sensorless second order sliding mode observer for induction motor, IEEE International Conference On Control Applications, 1933-1938, 2010.

F. Tahami, A. Shojaei and D. A. Khatir, A diversity based reconfigurable method for fault tolerant control of induction motors, International Symposium On Power Electronics, Electrical Drives, Automation and Motion, 66-71, 2006.

\section{Appendix}

The induction motor used in this work is a $1.5 \mathrm{KW}, \mathrm{U}=$ $220 \mathrm{~V}, 50 \mathrm{~Hz}, I_{n}=7.5 \mathrm{~A}$. The parameters are: $R_{s}=$ $1.633 \Omega, R_{r}=0.93 \Omega, L_{r}=0.076 H, L_{s}=0.142 H, L_{m}=$ $0.099 H, J=0.0111 \mathrm{Kg} \cdot \mathrm{m}^{2}, f=0.0018 \mathrm{~N} . \mathrm{m} / \mathrm{rad} / \mathrm{s}$ and $P=2$. 